\title{
Multilinguales
}

\section{La réduction de la valence verbale : le cas de la passivation en amazighe}

Reduction of Verb Valence: the Case of Passivization in Amazighe

\section{Lhassane Andam}

\section{OpenEdition}

12 Journals

Édition électronique

URL : http://journals.openedition.org/multilinguales/1002

DOI : $10.4000 /$ multilinguales. 1002

ISSN : 2335-1853

Éditeur

Université Abderrahmane Mira - Bejaia

Édition imprimée

Date de publication : 1 juin 2015

Pagination : 65-85

ISSN : 2335-1535

\section{Référence électronique}

Lhassane Andam, «La réduction de la valence verbale : le cas de la passivation en amazighe », Multilinguales [En ligne], 5 | 2015, mis en ligne le 01 juin 2015, consulté le 10 décembre 2020. URL: http://journals.openedition.org/multilinguales/1002; DOI : https://doi.org/10.4000/multilinguales. 1002

Ce document a été généré automatiquement le 10 décembre 2020.

\section{cc) (1) $९$}

Multilinguales est mise à disposition selon les termes de la Licence Creative Commons Attribution Pas d'Utilisation Commerciale - Pas de Modification 4.0 International 


\title{
La réduction de la valence verbale : le cas de la passivation en amazighe
}

Reduction of Verb Valence: the Case of Passivization in Amazighe

\author{
Lhassane Andam
}

1 Nous nous proposons dans cet article de procéder à la description des tours passifs en amazighe ${ }^{1}$ en général et plus particulièrement en tachelhite, variété dialectale en usage dans le Souss et le Haut Atlas au Maroc.

Le passage d'une construction active à une structure passive a une incidence syntaxique sur la relation entre le prédicat verbal et le nombre d'arguments que celuici appelle. L'insertion de la morphologie passive engendre la réduction de la valence ${ }^{2}$ verbale.

L'objectif essentiel est de rendre compte du paradoxe des rôles et des fonctions dans les constructions passives. L'approche que nous adoptons ici s'appuie essentiellement sur les critères développés dans Milner (1986), Cadi $(1994,2006)$ et Dik $(1989,1997)$, entre autres.

Les deux premiers chercheurs, qui s'inscrivent dans l'optique de la Grammaire Générative et Transformationnelle (GGT), préconisent une analyse transformationnelle basée sur la dérivation du tour passif à partir d'une structure active. Quant au dernier, qui n'est autre que le chef de l'école néerlandaise d'obédience fonctionnelle, il opte pour une analyse en termes de perspective.

2 Ce travail s'articule en trois axes. Dans le premier, nous allons présenter la morphologie passive et ses fonctions sans oublier le passif des verbes transitifs indirects et l'emploi intransitif des prédicats dits symétriques. Dans le second, nous essaierons de mettre l'accent sur le paradoxe des fonctions et des rôles lors de la transformation passive. Dans le troisième, le problème du passif sera reconsidéré dans une optique fonctionnelle où il est traité comme une inversion de la perspective sans pour autant qu'un processus transformationnel soit envisagé. 


\section{Le morphème du passif et ses fonctions}

3 En amazighe, la forme verbale passive est discernable par sa marque caractéristique, à savoir $t t$-. Soulignons que ce morphème peut varier et ce, en fonction du contexte de son apparition. Les formes essentielles sont les suivantes: $t t-$, $t t u-$, ttuy- et $t t y-$. Les contextes phoniques dont elles sont tributaires peuvent être déterminés ainsi :

i. les deux premiers allomorphes apparaissent généralement devant une forme verbale basique à initiale consonantique comme l'illustrent les exemples ${ }^{3}(1 \mathrm{a}-\mathrm{b})$ :

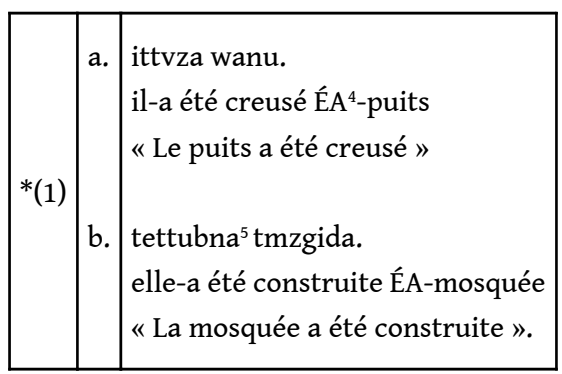

ii. les autres s'emploient quand le verbe est à initiale vocalique. Nous illustrons ces cas de figure par les exemples suivants :

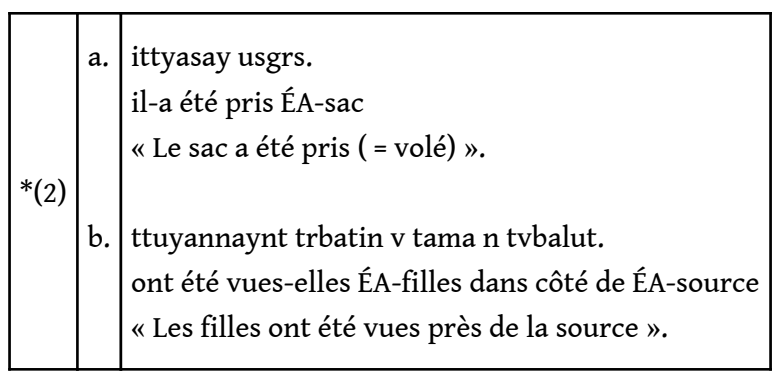

Il faut souligner que ces deux dernières variantes, en l'occurrence tty- et ttuy-, s'emploient indifféremment devant un verbe à initiale vocalique, mais la première forme est plus fréquente que la seconde.

4 L'incorporation des allomorphes $t t-, t t u$-, $t$ ty- et ttuy- à une tête verbale transitive fait que la phrase a une structure thématique passive. L'analyse de la transformation passive nous conduit d'abord à nous interroger sur la fonction de la particule du passif : "Le morphème de la passivation a deux fonctions: une fonction syntaxique et une autre sémantique ", précise Oussikoum (2013: 71).

5 Sur le plan syntaxique, le tour actif renferme généralement une tête verbale munie de deux arguments, en l'occurrence le sujet et le complément d'objet direct (COD) alors que dans la construction passive correspondante, le prédicat verbal passif est accompagné d'une seule dépendance thématique ${ }^{6}$, à savoir le sujet. Aussi faut-il souligner que le verbe passif réduit d'une unité le nombre de ses dépendances thématiques.

6 Au niveau sémantique, la structure active et sa correspondante passive expriment l'action et l'état résultant de cette action, respectivement. Les constructions passives se caractérisent essentiellement par la promotion de l'objet et l'élimination pure et simple 
du sujet sous-jacent, en l'occurrence l'agent. Considérons les tours (3a-b) en guise d'illustration.

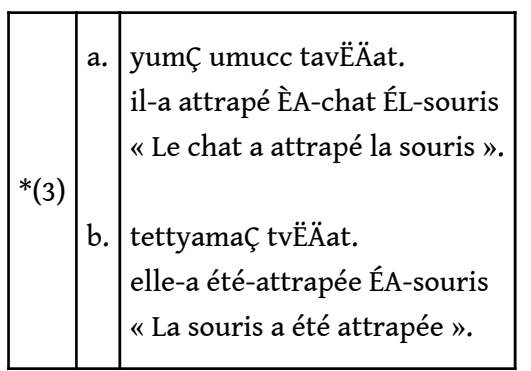

7 Dans (3a), nous avons la forme simple du verbe amÇ «prendre/attraper ». Ce dernier est un prédicat bivalent, en ce sens qu'il est accompagné de deux arguments, l'agentsujet umucc « le chat » et le patient-objet tavËÄat « la souris ».

En (3b), la forme verbale est dérivée par le biais de l'incorporation de la morphologie passive. Il s'ensuit que le patient devient le sujet de la phrase, l'accord avec le verbe prouvant cette fonction, et l'agent, c'est-à-dire le sujet du tour actif correspondant reste absent. En ce sens, le passif sous-entend un sujet indéterminé. Sur le plan syntaxique, le rôle de l'affixe du passif est de réduire d'un rang la valence intrinsèque d'un verbe (Tesnière, 1959).

En vue de préciser le rôle sémantique du morphème du passif, nous considérerons les exemples cités en $(4 a-b)$.

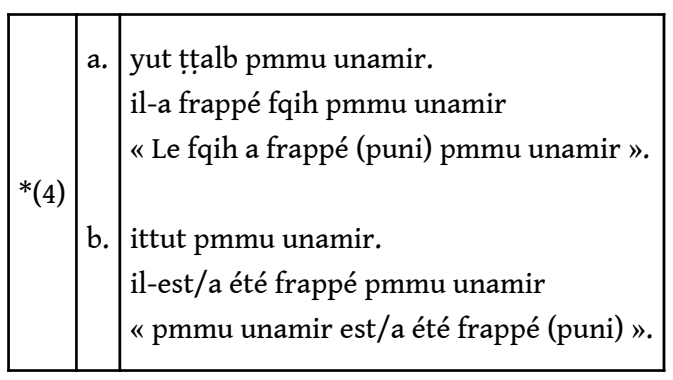

Dans l'énoncé (4a), le prédicat yut «il a frappé » a pour sujet IiÏalb «le fqih» qui représente « le participant actif du procès » (Oussikoum, $2013: 72$ ). Ce nominal partage les mêmes traits d'accord (nombre, genre et personne) avec l'indice de personne incorporé au verbe, à savoir $\mathrm{y}-\ll$ il ».

11 Dans la phrase passive correspondante (4b), l'affixe du passif traduit le processus tout en évinçant le sujet véritable.

L'indice de personne spécifié par les traits [+ masc.], [+sing.] et [+ $3^{\mathrm{e}}$ pers.] ne renvoie plus à l'agent, mais au participant passif pmmu unamir qui devient le sujet lexical explicite du prédicat passif ittut «il est/a été frappé ».

En principe, seuls les prédicats transitifs directs admettent la passivation. Cependant, les locuteurs amazighophones transposent au passif des constructions qui renferment des verbes transitifs indirects, à savoir les verbes suivis d'un complément d'objet indirect (COI) introduit par la préposition $i$ «à ». Considérons la séquence (5) qui 
comporte le prédicat ivrs «il a égorgé» qui est construit avec un syntagme prépositionnel et qui exprime un procès :

\begin{tabular}{|l|l|}
\hline *(5) & $\begin{array}{l}\text { ivËs pmad i tfunast. } \\
\text { il-a égorgé pmad à ÉA-vache } \\
\text { « pmad a égorgé la vache ». }\end{array}$ \\
\hline
\end{tabular}

Le tachelhite admet des constructions de type V + S + Prép. + O, dans lesquelles l'objet est indirect du point de vue de la syntaxe mais direct du point de vue de l'interprétation sémantique. Autrement dit, nous avons un prédicat transitif indirect vu que le verbe est séparé de son complément par un élément-ligature, ici la préposition $i$ «à ", mais du point de vue sémantique, ce syntagme prépositionnel désigne bien le patient de l'action comme s'il s'agissait d'un COD. La passivation de ce type de phrase est possible dans ce dialecte. Mais, elle entraîne forcément la suppression du fonctionnel $i$ « à » comme il est visualisé dans la construction (6). Ce qui n'est pas le cas en tamazighte des Ayt Mguild. En témoigne la structure (7) :

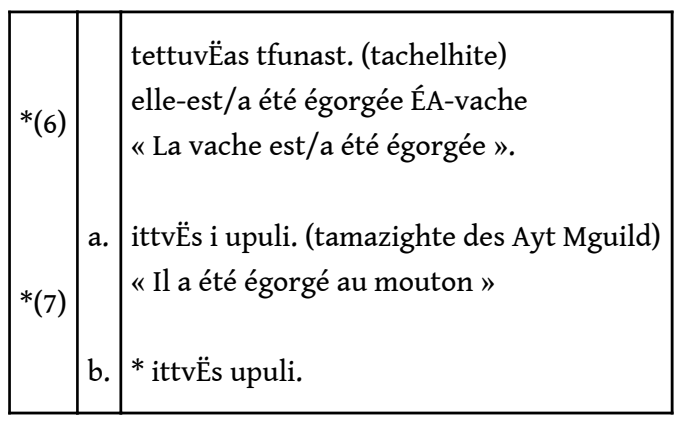

Dans Taïfi (2000), il est précisé, lors de l'analyse des constructions V + Prép. + N, que certains verbes transitifs indirects admettent contre toute attente la passivation, que le COI garde sa préposition dans la phrase passive (7a), que la suppression de $i$ « à » rend l'énoncé agrammatical $(7 b)$ et que la passivation est ressentie plus comme un tour impersonnel que comme une véritable passivation.

Notons que les structures de type Verbe + Prép. + Objet présentées et analysées par Taïfi (1995) ont été reprises par Sabia (1997). Ce dernier considère le passif des verbes transitifs indirects en amazighe comme un cas de transitivité inachevée. Nous ne mettons pas en doute le jugement d'acceptabilité d'une phrase telle que (7a) dans le parler des Ayt Mguild, mais ce dernier serait l'un "des îlots où le phénomène s'est le plus maintenu " (Ibid. : 13). La phrase (7b), jugée agrammaticale chez les Ayt Mguild, reste attestée dans l'aire tachelhitophone.

Le passif impersonnel évoqué supra existe bien dans d'autres parlers du Moyen Atlas tels le parler des Ayt Wirra. Nous l'illustrons par le tour (8) que nous empruntons à Oussikoum (Ibid. : 72) :

\footnotetext{
*(8) ittuffella i tcirratin

(Il a été entendu aux filles) «On a entendu les filles».
} 
D'après ce chercheur, le prédicat verbal ittuffella «il a été entendu » est accompagné d'un "sujet postiche», à savoir le morphème $i$ - «il» qui ne représente qu'un simple élément formel sans aucun statut sémantique.

Aussi suggère-t-il de considérer l'énoncé en question comme un exemple qui illustre le passif dit « impersonnel ».

Le préfixe intransitivant/passivant tt- est la marque spécifique du passif en amazighe. Il existe, cependant, un nombre important de verbes simples qui peuvent apparaitre dans des contextes où ils ont une valeur passive. Ces verbes, dits neutres ou symétriques, peuvent fonctionner comme :

a. verbes transitifs dans des énoncés bivalents où le premier argument est sujet et le second est $\mathrm{COD}$ :

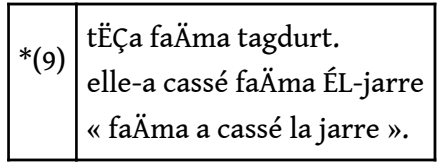

b. verbes intransitifs, le COD de l'énoncé précédent devient sujet- patient et le sujetagent se voit exclu :

*(10) tËÇa tgdurt.

elle-est/a été cassée ÉA-jarre

« La jarre est/a été cassée ».

L'emploi intransitif des prédicats verbaux dits réversibles est à rapprocher de la construction passive. Considérons les phrases suivantes:

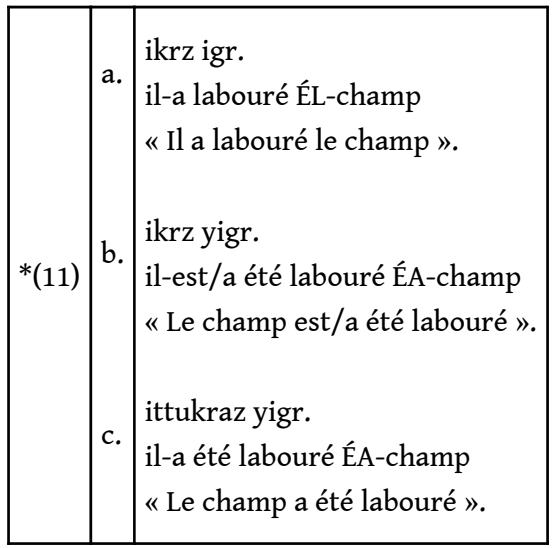

La seule différence observable entre les deux dernières structures concerne le prédicat verbal (11b) : le verbe apparaît sous sa forme simple et semble correspondre à (11c) dont le verbe est déterminé par l'incorporation du morphème du passif $t t-$.

Dans les deux cas, c'est le COD igr «le champ » [+ÉL] de l'énoncé actif (11a) qui est devenu le sujet syntaxique et le patient sémantique yigr [+ÉA]. (11b-c) illustrent la possibilité pour un prédicat verbal simple (à sens passif) de coexister avec la forme dérivée du verbe correspondant. À ce propos, Cadi (1987) souligne que «si le passif 
simple a recours à une forme dérivée, c'est qu'il n'a pas encore acquis son entière autonomie par rapport à l'actif» (p. 109).

Le problème auquel nous nous heurtons ici est de voir s'il faudrait considérer la forme verbale simple ikrz «il est/a été labouré » utilisée dans l'énoncé (11b) comme une véritable forme passive au même titre que la forme verbale dérivée de la construction (11c).

21 Il y a lieu d'être prudent, car répondre par l'affirmative nous conduira à admettre l'existence de deux passifs en amazighe. Nous préférons quand même maintenir la "valeur passive du verbe simple" sans pour autant postuler une équivalence et une correspondance parfaites entre les deux formes simple et dérivée. En effet, nous ne perdrons pas de vue qu'au niveau morphologique, la première forme est pareille à l'emploi transitif du verbe en question tandis que la seconde se caractérise par le préfixe $t t-$, l'indice spécifique du passif. Sur le plan sémantique, Chaker (1995) affirme que la forme simple exclut toute référence, directe ou indirecte, à l'agent alors qu'avec la forme préfixée, ce dernier est absent, mais reste quand même envisagé. La différence qui s'établit entre les deux formes, selon l'auteur (Ibid.), est celle qui existe entre :

a. un prédicat d'existence (le verbe simple) qui pose l'existence d'un procès-verbal, attribué au premier déterminant, et ;

b. un verbe dont le premier déterminant est explicitement posé comme un patient subissant un procès effectué par un agent extérieur, non mentionné. Il s'agit, selon la terminologie classique des sémitisants, d'un "passif à agent inconnu" [...]. Avec la marque du passif, l'agent est absent mais cependant envisagé alors que le verbe simple exclut toute référence, même vague ou implicite, à un actant extérieur » (p. 74).

Sans nous appesantir sur ce point, nous admettons que la véritable forme verbale passive est bien celle qui est discernable par sa marque caractéristique, à savoir le préfixe $t t$ - (ou l'une de ses variantes). Son incorporation à une tête verbale transitive fait que la phrase a une structure thématique et argumentale passive. Le rôle thématique du COD n'est plus saturé dans sa position canonique. La position de l'objet est remplie par une catégorie vide et la position de l'argument sujet est nonthématique. Les deux positions se trouvent alors étroitement corrélées. Il en découle un paradoxe des fonctions et des rôles (Cadi, 2006).

\section{La géométrie des rôles et des fonctions}

La définition du passif qui prévaut depuis toujours en grammaire traditionnelle et qui continue à s'imposer chez les générativistes est celle du "renversement » de l'actif: c'est une opération qui consiste à promouvoir le COD dans le site propre au sujet et à réduire ce dernier à un simple terme périphérique (complément d'agent) en français par exemple ou encore à l'effacer purement et simplement comme c'est le cas de la langue amazighe.

Il faut rappeler qu'en amazighe le passif ne repose pas sur un échange symétrique entre le sujet et le COD. La relation entre les constructions active et passive est de nature asymétrique : la passivation d'une phrase entraîne la conversion du COD en sujet et l'effacement du sujet de l'actif. L'amazighe ne dispose que d'un passif sans complément d'agent. Il semblerait qu'on perde un argument en cours de route - le sujet de l'actif -, ce qui rend malaisé l'analyse classique par renversement (Ibid.). 

que l'interprétation change. La passivation telle qu'elle a été définie supra consiste à effacer le sujet et à assurer le placement de l'objet dans la position réservée à l'argument indirect. Le prédicat verbal muni du marqueur $t t$ - se comporte alors comme un verbe intransitif, voire comme un prédicat inaccusatif'. La position de l'objet n'est plus un site où le cas objectif peut être assigné. À ce propos, Rouveret (1987) avance le principe suivant : «l'adjonction de la morphologie passive à la tête verbale V retire à cette tête la capacité d'assigner le Cas Objectif » (p. 210).

En amazighe, la catégorie $t t-V$ n'assigne pas le Cas Objectif, car l'affixe $t t$ - neutralise le trait [-N] retirant ainsi à $\mathrm{V}$ son statut de marqueur casuel. Devenant une position noncasuelle, la position de l'objet ne constitue plus un site approprié pour l'apparition d'un item lexical. Le nominal qui y figure en structure profonde la quitte obligatoirement et monte par conséquent dans la position occupée par le sujet où il sera marqué pour le Nominatif. Afin de mieux préciser les caractéristiques du passif, considérons les structures suivantes :

\begin{tabular}{|l|l|l|}
\hline$*(12)$ & a. & $\begin{array}{l}\text { mäln middn amttin. } \\
\text { ont enterré-ils gens ÉL-mort } \\
\text { « Les gens ont enterré le mort ». } \\
\text { b. } \\
\text { ittumÄal umttin. } \\
\text { il-a été enterré ÉA-mort } \\
\text { «Le mort a été enterré ». }\end{array}$ \\
\hline
\end{tabular}

Dans la construction (12a), le prédicat mäln "ils ont enterré » distribue les rôles thématiques agent pour l'argument indirect middn «les gens» et patient pour l'argument direct amttin « le mort ». Dans (12b), le verbe fléchi est morphologiquement à la forme passive. Sa passivation engendre la réduction du nombre d'arguments réalisés en surface, en ce sens que seul l'argument objet est réalisé dans la position consacrée au sujet après l'absorption du sujet véritable par la morphologie passive. Nous pouvons dire avec El Moujahid (1993) qu'en amazighe le passif «est de type exclusivement "inachevé". Le système ne connait pas de construction où l'argument sujet se réalise par stratégie d'adjonction prépositionnelle, comme en anglais, en français et en arabe moderne» (p. 378).

Compte tenu de l'échange asymétrique qui caractérise les tours actif et passif, il faut noter qu'en amazighe, les fonctions en jeu sont le sujet et le COD. Les deux constructions sont réalisées respectivement par deux configurations syntaxiques positionnelles: V-S-O et Verbe passif-S. Le sujet se présente comme une position obligatoire dans toute proposition en vertu du Principe de Projection Étendue. La deuxième fonction, à savoir celle de l'objet qui fournit le sujet du tour passif, est à son tour nécessaire quand on sait qu'elle intervient dans l'interprétation du passif.

Dans la structure (12b), le nominal umttin « le mort » occupe une position qui ne lui est pas naturelle. Il figure dans la position associée au premier argument du verbe. Le fait qu'il soit marqué pour le nominatif et qu'il s'accorde avec le prédicat montre clairement qu'il est localisé, en surface, dans la position occupée normalement par le sujet. Mais, il ne faut pas oublier qu'au niveau thématique, il dépend de la position de 
l'objet. C'est ainsi qu'il reçoit le rôle thématique de patient. L'item lexical en question reçoit son rôle sémantique, en structure profonde, dans la position de l'objet et son cas, à la suite du déplacement, c'est-à-dire en surface, dans la position où figure le sujet. Les deux positions remplies par le sujet et l'objet se trouvent de ce fait impliquées et corrélées.

La confrontation des structures active et passive (12a-b) permet de formuler le constat suivant: dans la première, la géométrie des rôles et celle des fonctions se correspondent tandis que, dans la seconde, elles ne coïncident pas. En termes plus clairs, nous pouvons dire que les termes middn «les gens » (12a) et umttin «le mort » (12b) ont la même fonction, en l'occurrence la fonction sujet. Cependant, ils n'ont pas le même rôle : dans le premier cas, nous avons l'agent alors que dans le second, il s'agit de la fonction sémantique de patient. Il faut souligner également que le constituant amttin «le mort » est COD dans (12a) tandis que dans (12b), il devient sujet de la phrase et prend la marque de l'ÉA. Mais, ce nominal porte toujours le même rôle en dépit de la différence des fonctions. Ainsi, l'analyse de Milner (1986) met en évidence que les fonctions syntaxiques (sujet et objet) ne se superposent pas aux rôles thématiques impliqués par le verbe. Notons que les fonctions sémantiques en question ne coïncident pas avec la place d'argument. Les propriétés des rôles "sont des propriétés positionnelles " (Milner, Ibid.:20), elles peuvent être identifiées par l'examen des seules propriétés observables dans des configurations positionnelles. À ce propos, nous faisons nôtres les propos de Milner qui considère le tour passif comme " un cas où la géométrie des rôles et la géométrie des fonctions sont différentes. Plus précisément, il n'y a pas d'argument matérialisé dans la position de complément d'objet. Pourtant, cet argument existe bien, avec ses propriétés de rôle, dans une autre position » (p. 20).

Dans sa tentative de donner une solution au problème du passif, la théorie $d u$ Gouvernement et $d u$ Liage $^{8}$ fait appel aux notions de «catégorie vide», et de « coindexation ». La phrase passive (13a) est représentée par (13b) :

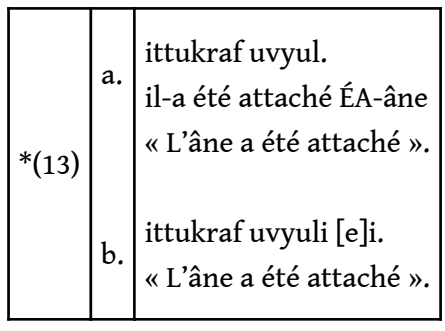

L'une des conséquences de l'adjonction du marqueur du passif à la tête verbale transitive est d'ôter à la position occupée par le sujet passif le statut de position thématique. Le verbe passif modifie le site de réalisation de l'argument direct. Dans l'exemple précédent, le nominal uvyul « l'âne » reçoit le rôle correspondant au COD du verbe krf "attacher", à savoir la fonction de patient qu'il supporte dans la phrase active. Afin de rendre compte de la distribution des rôles thématiques dans (13a), nous devons admettre qu'une position objet est présente aussi bien à l'actif qu'au passif, d'où la représentation (13b) et que le nominal figurant dans la position sujet reçoit son rôle thématique de cette position vide. Pour formaliser le lien qui unit les deux positions, nous nous servons de la coindexation comme l'illustre (13b). Dans cette configuration, la catégorie vide [e]i est co-indexée au nom uvyuli. Ce dernier reçoit son rôle de l'élément nul auquel il est associé. Le sujet du tour passif conserve donc les propriétés 
de la position coïncidente où se trouve la catégorie vide. La représentation (13b) constitue, de ce fait, une structure où «la non coïncidence et la coïncidence se lisent directement et simultanément " (Ibid. : 21).

Il reste maintenant à expliciter le statut de l'élément nul qui occupe la position de COD. C'est à sa faveur que cette dernière est récupérable. Comme nous l'avons souligné, le passif consiste en l'effacement du sujet actif et en la promotion de l'objet en position sujet. Il s'agit donc du déplacement d'un terme portant le rôle thématique de la position interne vers celle de l'argument indirect (le sujet), lequel déplacement doit entraîner en conséquence l'apparition d'une trace dans la position objet. Cette trace ne peut être identifiée selon le mécanisme proposé par Chomsky (1982) que comme une trace anaphorique assujettie au Principe $A$ du Liage ${ }^{9}$. Elle occupe une position argumentale et se trouve liée à un antécédent dans une position également argumentale. L'apparition de cet élément nul est dictée par le Principe de Projection. Dans les énoncés donnés en (13), le verbe krf «attacher » est sous-catégorisé pour un COD. Ledit principe stipule que ce verbe manifestera cette caractéristique dans toutes les structures grammaticales où il figurera.

L'examen des structures passives nous a permis de constater l'existence de deux stratégies assurant le marquage thématique d'un argument :

(i) soit il est réalisé dans une position thématique ;

(ii) soit il se trouve en relation formelle avec une position thématique.

Dans une phrase active, le COD reçoit son rôle de la position qu'il occupe. Dans une structure passive, il est réalisé dans un site non thématique (position sujet). Il doit alors être mis en relation avec la position objet vide. Le moyen approprié dont nous disposons pour opérer cette connexion est le concept de chaine. Cette notion se définit comme "la séquence de positions coindicées assurant la transmission d'un th-rôle d'une position thématique vide à un contenu [+argument] réalisé dans une position non-thématique " (Milner, 1986 : 48).

Ainsi, dans (13b), la séquence uvyuli, [e]i) est identifiable comme un argument discontinu reliant la position du COD et celle du sujet. Le nom uvyuli est marqué pour le Nominatif. La chaîne porte le th-rôle (rôle thématique) de patient correspondant à la position de la catégorie vide [e]i. Le sujet passif obtient ce th-rôle par l'appartenance à cette chaîne.

\section{Passivation ou inversion de la perspective}

En Grammaire Fonctionnelle (GF), les items lexicaux sont traités comme des prédicats. Ces derniers, qu'ils soient basiques ou dérivés ne sont pas considérés isolément. Ils font partie d'une structure ou d'un cadre prédicatif comportant les arguments qui leur sont associés.

Comme le souligne Jadir (2003), les règles de formation des prédicats entraînent généralement des changements dans la forme du prédicat-input et dans la structure des cadres prédicatifs. La passivation qui nous intéresse ici est un processus qui réduit la valence du prédicat par l'élimination de l'argument agent du prédicat basique. En nous référant à Dik (1997), nous formulons cette règle comme suit : 
\begin{tabular}{|l|l|}
\hline *(14) & $\begin{array}{l}\text { Règle de formation du prédicat passif } \\
\text { Input : Préd. [V] (x1)(x2) } \\
\text { Output : Préd. Passif [V] (--) (x2) }\end{array}$ \\
\hline
\end{tabular}

Nous donnons les phrases suivantes en guise d'exemples:

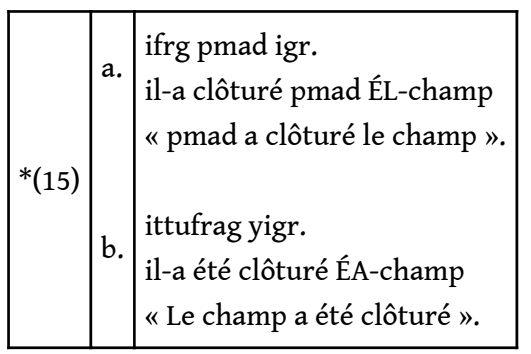

La construction (16b) met en évidence que la fonction sujet est assignée au terme qui fonctionne comme COD du prédicat input. Selon Dik (1989), le passif ne doit pas être vu comme une règle lexicale de détransitivisation, mais plutôt comme un phénomène syntaxique qui devrait être pris en charge par l'assignation de la fonction sujet. Quand celle-ci est assurée par l'argument agent, la phrase est à la voix active. Quant à la passive, elle est obtenue lorsque le sujet est un élément portant un rôle sémantique autre que celui de l'agent.

En GF, la fonction sujet est déterminée en termes de perspective. À la suite de Dik (1980), Moutaouakil (1987) en donne la définition suivante : «[La fonction Sujet est] assignée au terme pris comme étant le point de vue primaire de la perspective selon laquelle l'état de choses (ÉdC) exprimé dans la prédication est présenté»(p. 75). Considérons l'exemple donné en (16):

\begin{tabular}{|l|l|}
$*$ *(16) & $\begin{array}{l}\text { tçÇig iillu tafunast. } \\
\text { elle-a trait iïlu ÉL-vache } \\
\text { «iïlu a trait la vache ». }\end{array}$ \\
\hline
\end{tabular}

Dans cette clause, est exprimé un État de chose (ÉdC $\left.{ }^{10}\right)$, à savoir le fait que iilïu a trait la vache. Il est présenté selon le point de vue de l'agent auquel est assignée la fonction sujet, en l'occurrence iilïu. Il s'agit là du point de vue primaire de la perspective. Parler d'un point de vue principal suppose l'existence d'un point de vue secondaire. Ce dernier coïncide avec la fonction objet. En effet, dans l'exemple considéré, le nominal patient tafunast «la vache » est l'argument objet qui reste, de ce fait, une fonction « perspectivale » reléguée au second plan.

41 Dans les tours passifs, l'ÉdC est présenté à partir d'un point de vue différent de celui dont il est question dans le cadre prédicatif sous- jacent. C'est ce que nous entendons par l'inversion de la perspective. Nous présentons en (17) la construction passive correspondant à (16) : 
\begin{tabular}{|l|l|}
\hline$*(17)$ & $\begin{array}{l}\text { tettuÇÇig tfunast. } \\
\text { elle-a été traite ÉA-vache } \\
\text { « La vache a été traite ». }\end{array}$ \\
\hline
\end{tabular}

Dans cet énoncé, l'ÉdC est présenté suivant un point de vue secondaire, en ce sens que le terme qui assure la fonction sujet est en fait l'entité qui subit l'action, c'est-à-dire le patient-objet. Le véritable agent n'est pas mentionné. À ce niveau, nous adoptons la thèse de Jadir (Ibid.) qui considère que

la passivation implique une réduction du premier argument et un processus de 'changement d'argument' (argument shift), à travers lequel le second argument " usurpe » les droits $d u$ premier argument, i.e. sa position et sa fonction sémantique: l'Agent perd son statut d'argument et le Patient devient Procès. (p. 165 sq.)

En effet, l'agent perd sa position et sa fonction syntaxique. Le patient remplit cette position et fonctionne sur le plan syntaxique comme sujet. L'interrogation qui doit se poser ici est la suivante: qu'est-ce qui peut motiver le recours au passif? Pour y apporter quelques éléments de réponse, nous présentons quelques-unes des raisons avancées par Dik (1989) :

a. Quand la construction active comporte un sujet indéfini et un COD défini comme en (18a), il vaudrait mieux opter pour le tour passif (18b):

\begin{tabular}{|l|l|l|}
\hline$*(18)$ & a. & $\begin{array}{l}\text { ijdr yan unafal tigmmi n pmad. } \\
\text { il-a brûlé un fou ÉL-maison de pmad } \\
\text { « Un fou a brûlé la maison de pmad ». } \\
\text { b. } \\
\text { tettujdar tgmmi n pmad. } \\
\text { elle-a été brûlée ÉA-maison de pmad } \\
\text { «La maison de pmad a été brûlée ». }\end{array}$ \\
\hline
\end{tabular}

b. Lorsque l'agent n'est pas connu, non-identifié ou encore quand le locuteur désire volontairement le laisser comme tel, la priorité est donnée à la construction passive :

\begin{tabular}{|l|l|}
\hline$*(19)$ & $\begin{array}{l}\text { tettyagal tyddidt. } \\
\text { elle-a été accrochée ÉA-outre } \\
\text { «L'outre a été accrochée ». }\end{array}$ \\
\hline
\end{tabular}

Il s'avère alors que le recours au passif n'est pas arbitraire, mais découle d'un choix fait par le locuteur pour une raison donnée. En termes plus clairs, le locuteur ne transforme pas une structure active en une structure passive, mais choisit dès le départ de présenter un énoncé où le véritable sujet n'est pas mentionné. Une phrase comme (19) ne résulterait donc pas d'un processus transformationnel. Cela peut corroborer l'idée défendue par les fonctionnalistes, à savoir que la grammaire est dénuée de «mouvements » des constituants. Ainsi, un tour passif ne provient pas forcément d'une phrase dite active. La position dans laquelle figure un terme ne résulte pas de règles de déplacement comme le postulent les modèles transformationnels (GGT), mais de règles de placement. Le prédicat passif se comporte comme un verbe intransitif muni d'un seul argument réalisé en position sujet. 
$\mathrm{Au}$ terme de ce travail, nous soulignons avec Cadi (2006) que le passif est l'une des questions embarrassantes qui ont dérouté plus d'un linguiste dans toutes les langues où l'existence de valeur passive est admise. L'analyse de ce phénomène en amazighe esquissée ici nous a permis de tirer les conclusions suivantes :

\begin{tabular}{|l|l|}
\hline i. & le prédicat passif se reconnaît par l'affixe tt-; \\
\hline ii. & $\begin{array}{l}\text { en plus de verbes transitifs directs, certains prédicats transitifs indirects admettent la } \\
\text { passivation; }\end{array}$ \\
\hline iii. & l'emploi intransitif des verbes symétriques donne lieu à des structures à sens passif; \\
\hline iv. & $\begin{array}{l}\text { la promotion de l'objet en position sujet et la suppression de l'agent crée le paradoxe des rôles } \\
\text { et des fonctions; }\end{array}$ \\
\hline v. & $\begin{array}{l}\text { l'inversion de la perspective suivant laquelle l'ÉdC est présenté. Il est obtenu quand la fonction } \\
\text { sujet est assurée par un constituant autre que l'agent, en l'occurrence le patient. }\end{array}$ \\
\hline
\end{tabular}

Notons pour clore que, dans cet article, nous n'avons fait qu'effleurer le phénomène de la passivation en amazighe qui sollicite des recherches approfondies que nous inscrivons dans notre programme d'investigation.

\section{BIBLIOGRAPHIE}

ANDAM L., Chaînes thématiques en tachelhite, Doctorat $3^{\mathrm{e}} \mathrm{Cycle}$, Université Mohamed Ben Abdallah, FLSH, Dahr ElMehraz, Fès, 1997.

ANDAM L., Structure de la phrase simple et chaînes en amazighe : le cas du parler de Talgjounte (Taroudant), Thèse de Doctorat, Université Moulay Slimane, FLSH, Béni Mellal, 2013.

CADI K., Le système verbal rifain, forme et sens, Paris, SELAF, 1987.

CADI K., « Passif et moyen en berbère rifain », É.D.B. 12, Paris, 1994, pp. 107-117.

CADI K., Transitivité et diathèse en tarifite. Analyse de quelques relations de dépendances lexicale et syntaxique, Publications de l'Institut Royal de la Culture Amazighe, Série : Thèses et Essais - $\mathrm{N}^{\circ} 1$, Imprimerie El Maârif Al Jadida, Rabat, 2006.

CHAKER S., Linguistique Berbère : Études de syntaxe et de diachronie, Éd. Peeters, Paris-Louvain, 1995.

DIK S., Studies in Functional Grammar, New York, Academic Press, 1980.

DIK S., The Theory of Functional Grammar, Part. 1: The structure of the clause, Dordrecht, Foris Publications, 1997.

EL MOUJAHID L., Syntaxe du Groupe Nominal en Berbère Tachelhiyt (Parler d'Ighrem, Souss, Maroc), Thèse de Doctorat d'État. Université Mohammed V, Rabat, 1993. 
JADIR M., « Règles de formation de prédicats et paramètres textuels ", in Hermes, Journal of Linguistics $\mathrm{N}^{\circ}$ 30, 2003, pp. 159-177. Disponible sur: [download2.hermes.asb.dk/archive/ download/H30_08.pdf]

JADIR M., « Grammaire fonctionnelle (de discours) : Évaluation et perspectives », Hermes - Journal of Language and Communication Studies $\mathrm{N}^{\circ}-43,2009$, pp. 163- 201. Disponible sur :

[download2.hermes.asb.dk/archive/download/Hermes-43-7-jadir.pdf] MILNER J.-C., "Introduction à un traitement du passif », Groupe Grammaire Scientifique du Français D.R.L., Collection ERA, 642, Paris VII Jussieu, 1986.

MILNER J.-C., « Individualité linguistique et discontinuité morphologique », Recherches Nouvelles sur le Langage,D.R.L. Collection ERA, 642, Paris VII : 61-91, 1988.

MOUTAOUAKIL A., "VXS en arabe ", in Getting One's Words into Line: on Word Order and Functional Grammar. Functional Grammar Series 8 / NUYTS, J. \& de Schutter, G. (eds.), Foris Publications, Dordrecht, 1987.

OUSSIKOUM B., Dictionnaire Amazighe-Français. Le parler des Ayt Wirra, Moyen Atlas - Maroc, Publications de l'IRCAM, Rabat, (Éléments de grammaire, 2013, pp. 32-149).

PICABIA L., traduction de Some concepts and consequences of the theory of Government and Binding de N. Chomsky (1982) en La Nouvelle Syntaxe, Paris, Seuil, 1987.

PICA P., traduction de Lectures on Government and Binding de N. Chomsky (1981) en Théorie du Gouvernement et du Liage, Paris, Seuil, 1992.

ROUVERET A., Syntaxe et Dépendances Lexicales : identité et identification dans la théorie syntaxique, Thèse de Doctorat d'État, Paris III, 1987.

SABIA A., "Passif de verbes transitifs indirects en berbère : un cas de transitivité inachevée », in Voisinage Mélanges en hommage à la mémoire de K. Cadi, GREL 11, Publications de la Faculté des Lettres et des Sciences Humaines, 1997, pp. 121-139.

TAIFI M., « Verbes transitifs et passivation en tamazighte », communication à la Faculté des Lettres et des Sciences Humaines, Dahr El Mehraz, Fès le 13-04-1995.

TAIFI M., « De quelques verbes à préposition en tamazighte : contrainte sémantique et distribution syntaxique ", in Études berbères et chamito- sémitiques : mélanges offerts à Karl-G. Prasse, M.S.-15- Ussunamaziv, Peeters Press Louvain-Paris, 2000, pp. 317-332.

TESNIÈRE L., Éléments de syntaxe structurale, Klincksieck, Paris, 1959.

\section{NOTES}

1. Le terme amazighe renvoie ici à la langue amazighe en voie de standardisation au Maroc. Le tamazighte est la variété dialectale en usage au Moyen Atlas.

2. Selon Tesnière (1959), la structuration syntaxique est bâtie sur la connexion structurale et sémantique entre le terme régissant, ici le verbe considéré comme le pivot autour duquel gravite des satellites, et ses subordonnés. Ces derniers sont nommés " prime actant ", " second actant » et « tiers actant ». Ils renvoient au sujet, au complément d'objet direct et au complément d'objet indirect, respectivement. Ainsi, la valence peut désigner simplement le nombre maximal d'actants qu'un verbe peut recevoir. Rappelons que le vocable de valence est emprunté au vocabulaire de la chimie et renvoie au nombre de liaisons d'un atome avec d'autres atomes dans une combinaison. 
3. Il est à préciser que les exemples sont transcrits conformément à la notation latin- IRCAM. Signalons aussi que la majuscule n'est notée ni en début de phrase, ni à l'initiale des noms propres.

4. En amazighe, la majorité des noms masculins commencent par $a, u$ ou $i$ et les noms féminins par le segment $t$ suivi de l'une des voyelles précitées. Les noms caractérisés ainsi sont généralement sujets à la variation d'état. Cette appellation renvoie dans la littérature amazighisante à l'une des deux formes sous lesquelles un nom peut se présenter, l'une non marquée dite État Libre (ÉL) et l'autre marquée appelée État d'Annexion (ÉA).

5. Il s'agit là de l'un des rares cas où le schwa (le $e$ « muet ») est maintenu. Sa présence s'explique ici par la rencontre de trois consonnes identiques. Dans (1b), le premier $t$ est la marque flexionnelle de la $3^{\mathrm{e}}$ personne du féminin singulier et les deux autres représentent le morphème du passif.

6. L'étiquette de dépendance thématique, proposée par Chomsky (1981), renvoie à la notion d' argument. L'auteur souligne qu'il ne faut pas confondre dépendance thématique indirecte (le sujet) et dépendances thématiques directes (les compléments d'objet). Dans la même optique, Milner (1986) considère les compléments d'objet comme des arguments directs dans la mesure où ils font partie de la projection du verbe, ce qui n'est pas le cas pour le sujet dont la présence ne dépend pas seulement de la tête verbale, mais de la phrase dans son ensemble. Aussi est-il qualifié d'argument indirect du verbe.

7. Les verbes que les générativistes nomment « prédicats inaccusatifs » appartiennent à la classe des verbes intransitifs. Leur caractéristique essentielle est que l'unique argument qu'ils appellent est en fait complément d'objet en structure profonde et sujet en surface. Rentrent dans cette catégorie les verbes de mouvement tels $\mathrm{lkm}$ "arriver » et ddu "partir», et les verbes qui expriment une action sans agent comme $\ddot{\mathrm{E}}$ « tomber » et mmt « mourir ».

8. Après la Théorie Standard et la Théorie Standard Étendue, Chomsky a élaboré la Théorie des Principes et Paramètres dont les fondements théoriques et méthodologiques sont exposés dans l'ouvrage: Théorie du Gouvernement et du Liage (trad. Fr. 1992). Cette dernière s'appuie sur les principes et objectifs suivants :

- la grammaire est organisée autour de principes universels, lesquels principes sont présentés comme étant propres à l'espèce humaine ; ils permettraient l'acquisition du langage ;

- les paramètres définis par la théorie grammaticale caractérisent la manière dont les diverses langues appliquent ces principes; les paramètres permettent d'établir des différences et des analogies entre les langues, dans une perspective typologique ;

- la grammaire est définie comme un ensemble de modules autonomes (théorie du liage, théorie du gouvernement, théorie du cas, théorie des rôles thématiques, théorie du contrôle, etc.) ;

- la représentation linguistique est limitée à quatre niveaux: les structures profondes, les structures de surface, la forme phonétique, la forme logique.

9. Principe A : une anaphore est liée dans sa catégorie gouvernante. En d'autres termes, une entité anaphorique, étant dépourvue de référence intrinsèque, doit avoir un antécédent dans le domaine minimal qui la contient.

10. En GF, les prédications nucléaires expriment des ÉdC. Le terme ÉdC, écrit Dik (1989: 89, 1997a), est pris dans son sens large de « représentation de quelque chose qui peut avoir lieu dans un monde donné ». Quatre types d'ÉdC sont à distinguer, à savoir des Actions, des Procès, des Positions et des États exemplifiés respectivement dans les phrases suivantes (Jadir, 2009 : 164 sq., note 2) :

(i) a. Jean a giflé Lydie (Action).

b. La tempête a renversé le bateau (Procès).

c. Paul est debout (Position).

d. Catherine est livide (État). 


\section{RÉSUMÉS}

L'objet de cet article est le traitement de la passivation en amazighe en tant que processus réducteur de la valence verbale. Il nous sera ainsi permis de décrire les différents changements qui affectent la structure phrastique en passant de la diathèse active à la diathèse passive. Notre analyse inscrite, dans un premier temps, dans une optique transformationnelle mettra au jour le paradoxe des rôles et des fonctions qui caractérisent les tours passifs. Dans un second temps, nous présenterons une approche alternative où la passivation est vue comme un processus portant sur l'inversion de la perspective, laquelle approche d'obédience fonctionnelle dikienne de règles de placement qui n'impliquent aucune opération transformationnelle.

The purpose of this article is to describe the passive construction as a reducing form of verb valence in amazighe. Thus, we will be allowed to feature the various changes that affect the structure periphrastically, from the active diathesis to the passive diathesis. Our analysis reveals, on the one hand, with a transformational view the paradox of roles and functions that characterizes the passive form. On the other hand, we will introduce an alternative approach where the passive form is considered as a process involving the reversal of perspective, an approach which uses positioning rules that involve no transformational operation.

\section{INDEX}

Mots-clés : valence, passivation, amazighe, rôle/fonction, perspective

Keywords : valence, passivization, amazighe, role/function, perspective

\section{AUTEUR}

\section{LHASSANE ANDAM}

Faculté des Lettres et des Sciences Humaines Agadir - Maroc 